\title{
Biodegradable starch-based composites: effect of micro and nanoreinforcements on composite properties
}

\begin{abstract}
Thermoplastic starch (TPS) matrix was reinforced with various kenaf bast cellulose nanofiber loadings $(0-10 \mathrm{wt} \%)$. Thin films were prepared by casting and evaporating the mixture of aqueous suspension of nanofibers (NFs), starch, and glycerol which underwent gelatinization process at the same time. Moreover, raw fibers (RFs) reinforced TPS films were prepared with the same contents and conditions. The effects of filler type and loading on different characteristics of prepared materials were studied using transmission and scanning electron microscopies, X-ray diffractometry, Fourier transform infrared spectroscopy, thermogravimetric analysis, differential scanning calorimetry, and moisture absorption analysis. Obtained results showed a homogeneous dispersion of NFs within the TPS matrix and strong association between the filler and matrix. Moreover, addition of nanoreinforcements decreased the moisture sensitivity of the TPS film significantly. About $20 \%$ decrease in moisture content at equilibrium was observed with addition of $10 \mathrm{wt} \% \mathrm{NFs}$ while this value was only $5.7 \%$ for the respective RFs reinforced film.
\end{abstract}

Keyword: Thermoplastic starch (TPS) matrix; Nanofibers (NFs) 